\title{
Complete Analysis of Multireflection and Spectral-Shadowing Crosstalks in a Quasi-Distributed Fiber Sensor Interrogated by OFDR
}

\author{
Kivilcim Yüksel, Member, IEEE, Véronique Moeyaert, Member, IEEE, Patrice Mégret, Member, IEEE, and \\ Marc Wuilpart, Member, IEEE
}

\begin{abstract}
We present the analysis of a quasi-distributed fiber sensor based on the concatenation of identical low-reflective fiber Bragg gratings (FBGs) taking into account both multireflection and spectral-shadowing crosstalks. This allows obtaining more realistic values of the design parameters such as the maximum number of sensing points, the reflectivity of the gratings, and the distance between the sensing points.
\end{abstract}

Index Terms-Fiber Bragg gratings (FBGs), fiber optics sensors, interferometry, optical frequency domain reflectometer (OFDR).

\section{INTRODUCTION}

$\mathbf{O}$ PTICAL fiber optic sensors represent a powerful class of alternative technologies to the conventional electrical sensors thanks to their low-weight, small dimensions, and immunity to electromagnetic interferences. Among all fiber optic sensors, those based on fiber Bragg gratings (FBG) have a significant potential and have been developed for a wide variety of mechanical sensing applications including monitoring of civil structures (highways, bridges, buildings, dams, etc.), smart manufacturing and nondestructive testing (composites, laminates, etc.), remote sensing (oil wells, power cables, pipelines, space stations, etc.), smart structures (airplane wings, ship hulls, buildings, sports equipment, etc.), as well as traditional strain, pressure and temperature sensing [1]. The global market size of FBG sensors was reported in the range of $15 \mathrm{M}$ to $35 \mathrm{M}$ dollars USD a year, with an annual growth rate of $15 \%$ to $25 \%$ [1]. This growth is mainly due to the various advantages of the gratings. They are low-cost, mass producible devices providing self-referencing and wavelength-encoded linear response. Furthermore, these devices allow high degrees of multiplexing (wavelength-, time- or spatial-division) and, therefore, enable multipoint sensing schemes where many gratings can be placed within a single optical fiber.

Manuscript received March 15, 2011; revised June 17, 2011 and July 20, 2011; accepted July 22, 2011. Date of publication September 12, 2011; date of current version April 06, 2012. This work was supported in part by the Fonds de la Recherche Scientifique-Crédit aux Chercheurs (FNRS) and in part by the Interuniversity Attraction Pole IAP 6/10 program of the Belgian Science Policy. The associate editor coordinating the review of this paper and approving it for publication was Dr. M. Nurul Abedin.

The authors are with the Department of Electromagnetism and Telecommunications, Faculté Polytechnique, University of Mons, Mons, 7000 Belgium (e-mail: kivilcim.yuksel@umons.ac.be).

Color versions of one or more of the figures in this paper are available online at http://ieeexplore.ieee.org.

Digital Object Identifier 10.1109/JSEN.2011.2167142
Wavelength-division multiplexed (WDM) sensors are the most commercially encountered systems, which permit to address about ten gratings on a single fiber [2]. In these systems, a unique range of operating wavelength (under identical strain and temperature conditions) is dedicated to each grating of the concatenation. Therefore, the number of sensing points is directly limited by the spectral ranges of the source and the detector, and the wavelength spacing between two gratings which depends on the range of the physical parameter to be measured.

In order to overcome these limitations, several solutions based on an Optical Time Domain Reflectometer (OTDR) have been proposed [3], [4]. Recently, two quasi-distributed temperature sensors based on wavelength-tunable OTDR which interrogates 90 FBGs with $1{ }^{\circ} \mathrm{C}$ of accuracy on the measured temperature were reported [5], [6]. The main challenges of these sensors are related to the inevitable dead-zone associated with the OTDR detection scheme (which in turn limits the minimum physical distance between sensing points), and to the requirement for highly expensive equipment (i.e., tunable OTDR). Moreover, the measurement time needed to interrogate the whole system varies between a few minutes to a few tens of minutes [5], [6].

Optical frequency domain reflectometer (OFDR) providing millimetre resolution over medium measurement ranges (up to a few hundreds of meters) has also been attracting great attention as an interrogating tool for several sensor applications. Use of FBG arrays interrogated by OFDR for strain sensors were studied and experimentally demonstrated [8]-[10], [12]. In these demonstrations, however, the distortions inherent to the concatenated FBG array were included, neither in the calculations nor for the analysis of the experimental results. Therefore, limitations of the technique due to these distortions were not evaluated.

In this paper, we describe a fiber sensor system for temperature measurement using as sensing points a set of uniform, identical, low-reflective fiber Bragg gratings written in a single fiber and using an OFDR as the interrogator tool. In such a sensor, multireflection and spectral-shadowing crosstalk effects are of paramount importance. It was demonstrated in the literature that FBGs can be seen within a OFDR trace [10], [12] but the error on the demodulated spectrum due to these parasite effects has never been studied.

In this context, we analyse these parasite effects by the way of simulations. Moreover, practical solutions are proposed in order 


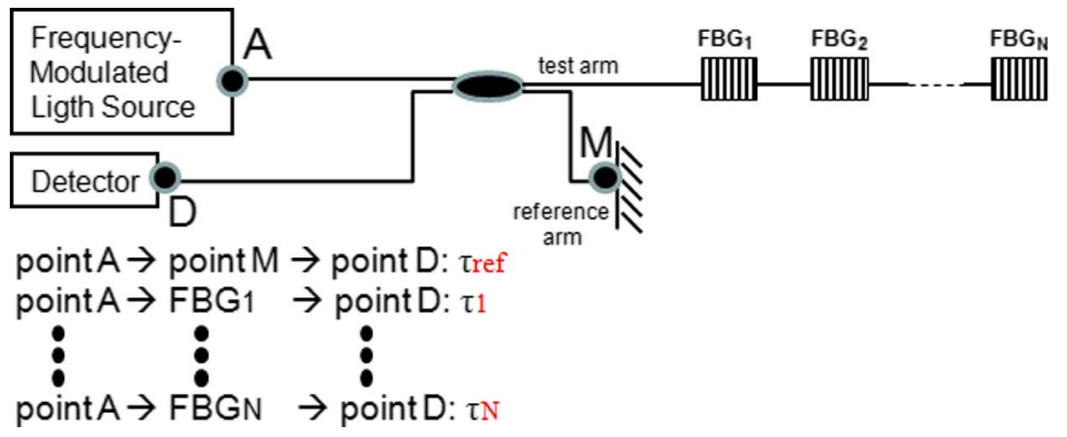

Fig. 1. Basic OFDR sensor interrogation setup with a series of concatenated FBGs.

to deal with these crosstalk effects. Simulations show that, the effect of multireflection crosstalk can be considerably avoided by using nonequal distances between sensing points. Indeed, for a signal to multireflection crosstalk ratio (SMRR) better than 10, the maximum number of FBGs that can be concatenated is enhanced from about 30 (equal distance case) to about 100 by using a uniform distribution of the distance between sensing points. The influence of spectral-shadowing crosstalk is found to be dependent on the following factor: the temperature deviation between the mean value of all downstream FBGs and the temperature to be measured by the FBG under test. By using an enhanced algorithm, the error on the measured temperature due to spectral-shadowing crosstalk (about $5{ }^{\circ} \mathrm{C}$, for $100 \mathrm{FBGs}$ ) has been considerably reduced (up to $3.5^{\circ} \mathrm{C}$ of enhancement).

To the best of our knowledge, this is the first time that the theoretical model takes into account both of these distortions to obtain more realistic values of the design parameters (i.e., maximum number of sensing points on a single fiber, reflectivity of the gratings, distance between the sensing points...). Solutions based on new system configuration (nonequal distances between FBGs) and enhanced algorithms were also proved to increase the maximum number of sensing points, still keeping an error on the measured temperature smaller than $1.5^{\circ} \mathrm{C}$.

This paper is organized as follows: in Section II, the principles of the quasi-distributed temperature sensing are explained. The parameters as well as the performance limits of our technique are then analysed in Section III taking into account the main distortions present in the system, namely multireflection crosstalk and spectral-shadowing crosstalk. We focus on these potential issues by the way of simulations. Finally the main conclusions of the work are reported in the last section.

\section{SENSOR PRINCIPLE}

The presented sensor is based on the association of two technologies: fiber Bragg grating (FBG) and Optical Frequency Domain Reflectometry (OFDR). Gratings act as sensing points whereas the OFDR is used as the interrogating device.

In its simplest form, a fiber Bragg grating is a permanent and periodic modification of the core refractive index value along an optical fiber. A fiber Bragg grating is described by its length $L$ over which the variation on the refractive index is realized, the periodicity $\Lambda$, and the amplitude of the refractive index modulation $\delta n$.
The inscription of the perturbation in the fiber core refractive index induces mode coupling between two counter propagating beams. That coupling occurs for the resonance wavelength of the grating, the Bragg wavelength, obtained by

$$
\lambda_{\text {Bragg }}=2 n_{\text {eff }} \Lambda
$$

where $n_{\text {eff }}$ is the effective refractive index of the core at the Bragg wavelength $\lambda_{\text {Bragg. }}$.

Due to the thermal dependence of the refractive index and the thermal expansion coefficient of the optical fiber, the Bragg wavelength is shifted toward longer wavelengths by temperature. The typical order of magnitude of temperature sensitivity of the Bragg wavelength around $1550 \mathrm{~nm}$ is of $10 \mathrm{pm} /{ }^{\circ} \mathrm{C}$ without spectral shape modification and without hysteresis [2].

OFDR (Optical Frequency Domain Reflectometry) is a technique for high-resolution metrology that can find applications in both optical fiber sensors and telecommunication networks [11]. Fig. 1 shows the block diagram of the OFDR interrogating cascaded FBGs.

In its basic configuration, the optical carrier frequency of a tunable laser source (TLS) is swept linearly in time without mode hops. Then, the frequency-modulated continuous-wave signal (probe signal) is split into two paths, namely the test arm and the reference arm (see Fig. 1). The former includes the device under test (DUT) whereas a reference reflector (also called local oscillator) is placed in the latter. The test signal which is reflected back from the reflection sites in the test arm coherently interfere at the coupler with the reference signal returning from the reference reflector. Superposition of the interfering signals is converted into electrical domain by the detector which yields the beat terms that are related to the optical amplitude and phase responses of the reflection sites in the DUT. In order to observe these beat terms, the photocurrent at the detector output is sampled and Fourier transformed into frequency domain where the beat terms appear as peaks. Using a linear optical frequency sweep, the frequency domain can be mapped into a distance scale (the proportionality factor between beat frequency and the corresponding distance is determined by the rate of change of the optical frequency), while the squared magnitude of the signal at each beat frequency reveals the reflectivity of each reflection site.

As shown in Fig. 1, the probe signal launched into the system is divided into two paths and simultaneously propagates along both the test arm and the reference arm. The total round-trip 


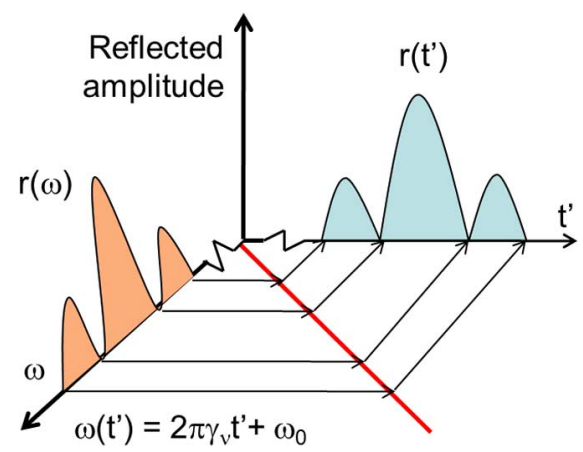

Fig. 2. Amplitude reflection coefficient is a function of optical frequency and can also be represented as a function time as the frequency of the TLS is swept linearly in time ( $\gamma \nu$ constant $)$.

time of the reference signal's path $(\mathrm{A} \rightarrow \mathrm{M} \rightarrow \mathrm{D})$ is defined as $\tau_{\text {ref }}$. In the same way, the total round-trip time of the test signal is defined as $\tau_{i}$ for the FBG \#i.

The reflected light from each FBG in the concatenation, interferes with the reference light from the reference mirror. The global effect is the generation of a composite signal at the photodetector which includes the sum of the responses from all the FBGs and can be analysed by using the two-beam interference method. The AC part of the interference signal detected at the photodiode can be expressed as

$$
\begin{aligned}
u(t) \propto 2 \sigma E_{0}^{2} \sum_{i=1}^{N} \kappa_{i} a_{i}[\omega(t)] & \\
& \cdot \cos \left[2 \pi \gamma_{\nu}\left(\tau_{i}-\tau_{r e f}\right) t+\Phi(\omega(t))+\varphi\right]
\end{aligned}
$$

where $\sigma$ is a constant that depends on the photodetector sensitivity, $E_{0}$ is the amplitude of the probe signal's electric field, $\kappa_{i}$ is a constant representing all the fiber attenuation and coupling losses for the FBG \# $i, N$ is the number of FBGs in the concatenation, $\omega(t)$ is the angular frequancy of the probe signal, $\varphi$ is a constant (which is a function of initial phase, $\tau_{\text {ref }}, \tau_{i}[7]$ ), $a_{i}[\omega(t)]$ and $\Phi_{i}[\omega(t)]$ are, respectively, the amplitude and phase of the complex amplitude-reflection factor of each FBG. This function $r_{i}[\omega(t)]$ can be represented as

$$
r_{i}\left[\omega\left(t^{\prime}\right)\right]=a_{i}\left[\omega\left(t^{\prime}\right)\right] \exp \left[j\left(\Phi\left(\omega\left(t^{\prime}\right)\right)\right]\right.
$$

where $t^{\prime}$ is an independent variable. This reflection factor is a function of optical frequency, $\omega$ and can be mapped from optical frequency scale to time scale under the condition that frequency of the laser source is swept linearly in time as schematically represented in Fig. 2.

It can be seen from (2) that the amplitude of the reflection spectrum of each FBG, $a_{i}[\omega(t)]$ modulates a sinusoidal function with a unique beat frequency $\left(f_{b i}=\gamma_{\nu}\left(\tau_{i}-\tau_{\text {ref }}\right)\right)$. Therefore, when Fourier transform of the (2) is taken to obtain the OFDR trace, discrete reflections of this trace are related to the Fourier transforms of the FBG reflection spectra.

If a temperature change is applied on one of the FBGs in the concatenation, for instance on the $\mathrm{FBG} \# N$, the reflection spectrum of the FBG $\# N$ will be shifted by $\triangle \lambda$. Hence, $r_{N}[\omega(t)]$ will become $r_{N}[\omega(t)-\triangle \omega]$. As the laser frequency is linearly swept, this temperature-induced spectrum shift $\Delta \omega$, $\left(\triangle \omega=2 \pi c\left(\triangle \lambda / \lambda^{2}\right)\right)$, can be expressed in terms of an equivalent time shift, $\tau_{\text {DUT }}$ which is given by

$$
\tau_{\text {DUT }}=c \frac{\triangle \lambda}{\lambda^{2}} \gamma_{\nu}^{-1} \text {. }
$$

As stated before, Fourier transform of the reflection spectrum of FBG $\# N$ is found around the beat frequency $f_{\mathrm{bN}}$, $\left(f_{\mathrm{bN}}=\gamma_{\nu}\left(\tau_{N}-\tau_{\mathrm{ref}}\right)\right)$. Due to the time-delay property of Fourier analysis, the amount of shift in the time domain $\left(\tau_{D U T}\right)$ is equivalent to a shift in the phase slope in the frequency domain, i.e., $r_{i}\left(t-\tau_{\text {DUT }}\right) \leftrightarrow R_{i}(f) \exp \left(-2 \pi j f \tau_{\text {DUT }}\right)$ and the reflection spectrum of each FBG can be obtained by band-pass filtering the signal in the frequency domain around each beat frequency with a sufficient bandwidth. Then, inverse fast Fourier transform (IFFT) on this selected portion, can be used to recover the complex reflection spectrum of each grating independently from the others [12].

One should note that, after IFFT process, reflection coefficients of the FBGs can be mapped from time scale to the optical frequency scale as schematically represented in Fig. 2. Finally, by using the calibration characteristics, the temperature is deduced for each FBG in the network.

Note that, when a sufficient wavelength range is swept by the TLS inside the OFDR, analysis of only one OFDR trace is enough to interrogate the temperature profile of the whole array. The optical wavelength range to be swept depends also on the temperature range to be measured.

\section{SIMULATIONS AND DisCUSSION}

\section{A. Multireflection Crosstalk}

Multiple reflections from cascaded sensing points will influence the performance of the sensor. Our objective is to evaluate how the maximum number of grating elements that can be interrogated by the sensor is affected by the amount of multireflection crosstalk.

We studied this problem considering only the 3-reflection component (the power contribution of the 5-reflection component is smaller than $1 \%$ of the 3 -reflection contribution for the FBG reflectivity values smaller than $10 \%$ ). The sensor in the test arm consists of $\mathrm{N}$ cascaded sensing points (FBGs) with identical power reflectivity, $r$. The total length of the sensor is $L$. To be able to analyse the contributions of 3-reflection components on the signal, we consider all the possible paths giving the same round-trip time as the useful signal (they lead to the same beat frequency with the useful signal). These paths (considering that the useful signal is the reflection from FBG $\# N$ ) are schematically represented in Fig. 3 for the worst case where the FBGs are equally spaced. In this worst case analysis, we first calculate the total number of possible paths, $N_{p 3}$, which (after 3 reflections) results in the same path-length as the useful signal.

The total number of such combinations when the first reflection occurs at FBG \#(N-1) is equal to $(N-2)$. These combinations are schematically represented in Fig. 3 (blue lines). If the first reflection takes place at FBG \#(N-2), there will be $(N-3)$ possible cases giving the same round-trip distance. In the same way, if the first reflection occurs at the second FBG, there is only one possible way (represented as red line in Fig. 3) 


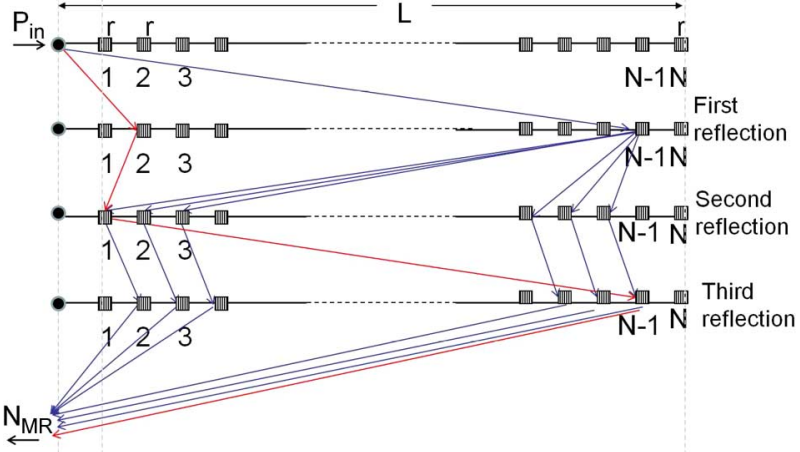

Fig. 3. Schematic representation of the possible paths for 3-reflection components for the worst case where the FBGs are equally spaced.

to have a total path length of $2 L$. As a result, $N_{p 3}$ can be expressed as

$$
N_{p 3}=\frac{(N-1)(N-2)}{2} .
$$

We can then formulate the power of the signal resulting from multiple reflection crosstalk which is superimposed on the useful signal, $N_{M R}$ as

$$
N_{M R}=P_{i n} N_{p 3} r^{3}(1-r)^{2 N-4} \exp (-\alpha 2 L) .
$$

where $P_{i n}$ is the optical power injected into the sensor (test arm of the OFDR), $r$ is the reflectivity of each FBG, $\alpha$ is the power attenuation coefficient in $\mathrm{m}^{-1}$, and $L$ is the length of the sensor from input to the position of FBG \#N in meter. Note that the light passes through $(2 N-4)$ FBGs in transmission for each 3-reflection path.

The power of the useful signal reflected from the FBG under test can be expressed as

$$
P_{S}=P_{i n} r(1-r)^{2(N-1)} \exp (-\alpha 2 L) .
$$

Finally, by using the expressions developed for the useful signal and the multireflection crosstalk contribution, the signal to multireflection crosstalk ratio (SMRR) of the sensor can be obtained as

$$
S M R R=\frac{P_{S}}{N_{M R}} .
$$

In addition to the above analytical analysis of the worst case where the FBGs are equally spaced, we conducted simulations for another case, the uniform random distribution of the distance between two successive FBGs. The path-lengths of all the 3-reflection components are first calculated for the random (uniform) distribution. The number of path-lengths which are within a tiny interval (defined by the spatial resolution of the OFDR) of the useful signal path-length gives the value of $N_{p 3}$. Finally, SMRR values are calculated as a function of reflectivity $r$ for both cases. Parameters of the distributions used in the simulations are summarized in Table I.

Comparison between these 2 cases for 50 concatenated gratings $(N=50)$ is shown in Fig. 4. As expected, use of the uniform distribution provides better performance than the equally spaced sensing points. When the perturbation due to
TABLE I

Studied Cases for the Distances Between Successive FBGs

\begin{tabular}{|c||l|}
\hline Equal distance & Distance $=20 \mathrm{~cm}$ \\
\hline Uniform distribution & $\begin{array}{l}\text { Minimum distance }=10 \mathrm{~cm}, \\
\text { Maximum distance }=30 \mathrm{~cm}\end{array}$ \\
\hline
\end{tabular}

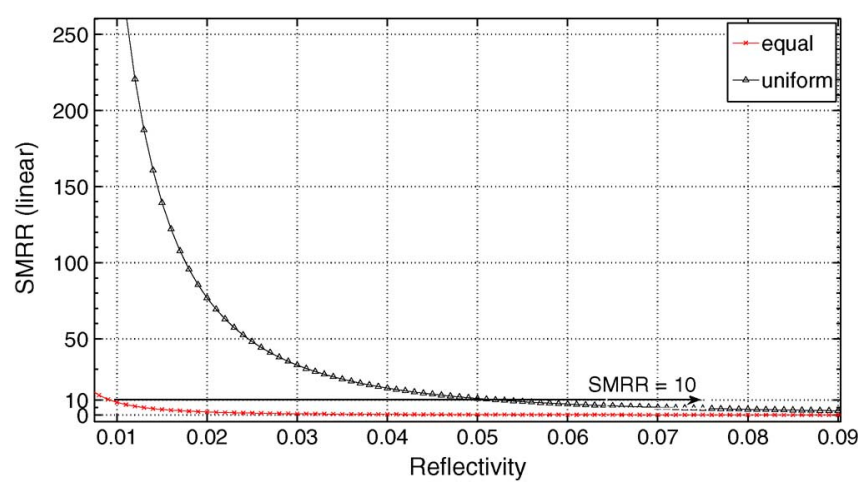

Fig. 4. Signal-to-multireflection crosstalk ratio (SMRR) versus FBG reflectivity $(r)$ for two different distributions of distances between the sensing points, $(N=50)$.

3-reflection crosstalk has an intensity of $10 \%$ of the useful signal $(S M R R=10)$, reflectivity values should be smaller than $5 \%$ for the uniform distribution case. This SMRR performance, however, cannot be achieved by equally spaced sensing points for any reflectivity value.

For different $\mathrm{N}$ values, Fig. 5(a)and (b) shows the SMRR versus reflectivity for the equal-distance, and uniform distribution respectively with Table II parameters.

For the equal distance case, in order to obtain a SMRR better than 10 , one can use up to about 30 FBGs with the reflectivity lower than 1.6\% [see Fig. 5(a)]. The number of FBGs can be substantially increased by using the uniform distribution as represented in Fig. 5(b). In the uniform distribution case, the reflectivity value required to obtain SMRR better than 10 is relaxed to $7.5 \%$ for $\mathrm{N}=30$. In addition, 100 FBGs can be interrogated by using reflectivity values smaller than about $3 \%$ and still having SMRR better than 10. As a result, use of random distribution (i.e., uniform) for the distance between the FBGs is proven to be a very efficient method to overcome the problem of multireflection crosstalk. Such distribution profile can be practically implemented by using recent automated FBG fabrication processes.

Finally we analyzed the effect of 5-reflection components on the SMRR values. In our extended code, we are able to calculate the number of all the possible 5-reflection components, then we select those whose have the same path-length as the useful signal for a given distribution (e.g., uniform). As an example, $N_{p 5}$ (the paths giving the same round-trip time as the useful signal after 5 reflections) has been calculated for $\mathrm{N}=100$. Fig. 6 shows a comparison between the case where both the 3- and 5-reflection components are taken into account and the case only 3-reflection components are considered. Very small decrease is observed due to 5 -reflection components.

\section{B. Spectral-Shadowing Crosstalk}

Spectral-shadowing crosstalk occurs when a concatenation of gratings sharing the same spectral characteristics are ad- 

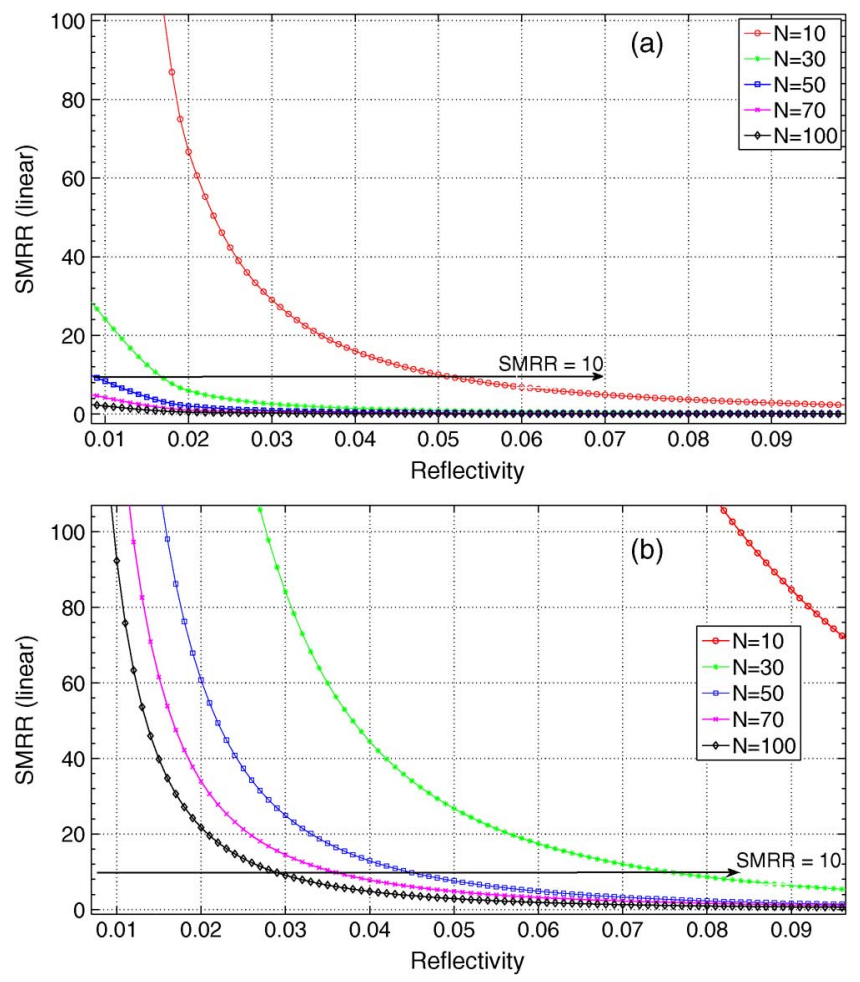

Fig. 5. SMRR versus reflectivity $r$ with different numbers of FBGs for (a) equal distribution, (b) uniform random distribution of the sensing points by Table II parameters.

TABLE II

Statistics on Spectral-Shadowing Crosstalk Simulations: MEAN AND STANDARD DEVIATION OF THE ERror, ON 1000 SAMPLES, FBG Under TeSt $(\# N)$ IS AT $40{ }^{\circ} \mathrm{C}$

\begin{tabular}{|c||c|c|}
\hline $\begin{array}{c}\text { Number of } \\
\text { concatenated gratings }\end{array}$ & $\begin{array}{c}\text { Mean value of the error } \\
{[\mathrm{pm}]}\end{array}$ & $\begin{array}{c}\text { Standard deviation } \\
{[\mathrm{pm}]}\end{array}$ \\
\hline \hline 10 & 3.25 & 0.62 \\
\hline 30 & 7.34 & 1.13 \\
\hline 50 & 9.79 & 1.49 \\
\hline 70 & 11.71 & 1.67 \\
\hline 100 & 14.23 & 1.87 \\
\hline
\end{tabular}

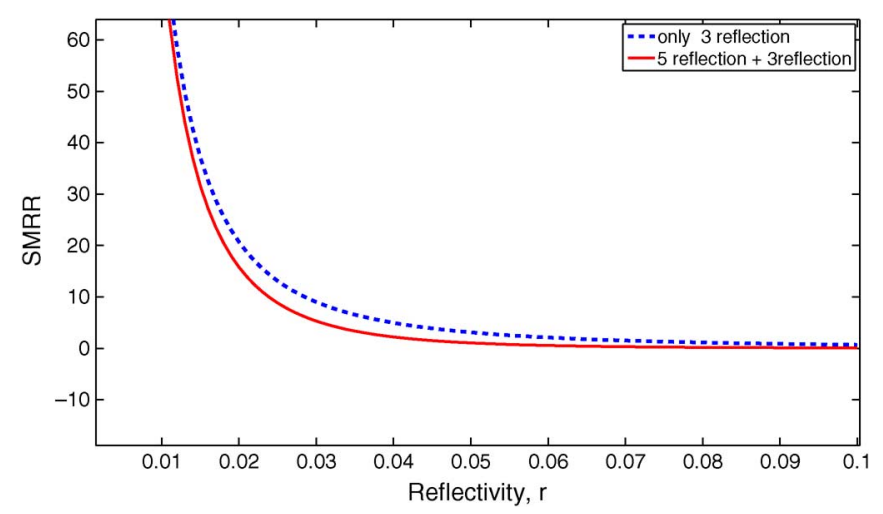

Fig. 6. SMRR versus reflectivity $r$ for $N=100$.

dressed simultaneously. Distortion occurs in the downstream FBG's spectra due to light having to pass twice through an upstream FBG [13]. This problem can be avoided by associating a coupler to each grating instead of serially connecting them

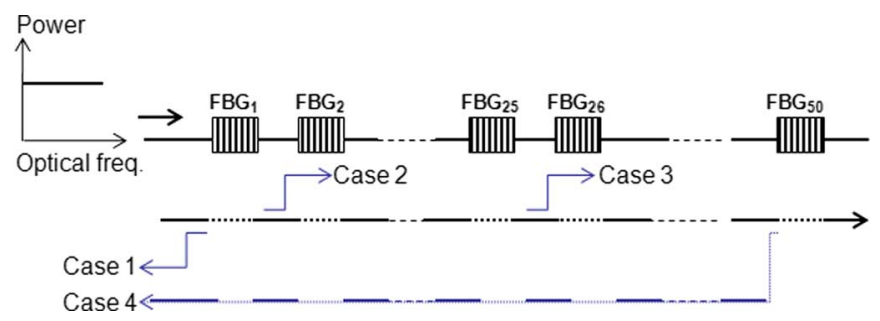

Fig. 7. Analysis of the light signal at different positions along the sensor.

at the expense of additional components and loss of power (insertion loss of the additional components).

Let us consider a concatenation of 50 FBGs. Obviously, the signal entered into the concatenation gets modified during its round-trip propagation along the fiber. Indeed, the spectrum of the light in front of each grating depends on the previous ones as their transmission spectra are superimposed. Therefore, one can already predict that the further the FBG under test, the more distorted the measured reflection spectrum. In order to visualize this effect, we analyse four cases shown in Fig. 7, which are representing the light signal at four different positions along its propagation through the concatenation, either in reflection (Case 1 and Case 4) or in transmission (Case 2 and Case 3). In this analysis, all FBGs are assumed to be at room temperature and to have about identical spectral characteristics (Bragg wavelength around $1583.7 \mathrm{~nm}$ at room temperature and reflectivity below $10 \%$ ) with small fluctuations in the central (Bragg) wavelength. These inevitable fluctuations due to fabrication process correspond to the specifications of the real gratings written in UMONS clean room facilities and can be modeled by a Gaussian distribution of the Bragg wavelengths with a standard deviation of $50 \mathrm{pm}$. In the simulations, $22{ }^{\circ} \mathrm{C}$ corresponds to room temperature.

Simulation results shown in Fig. 8 clearly demonstrate the modifications in power and shape of the transmitted and reflected spectra. Because of the shape deterioration of the reflection spectrum [see Fig. 8(a), case 4], determination of Bragg wavelength, the basis of temperature measurement, becomes difficult in the presence of the non-negligible spectral-shadowing crosstalk effect.

In its simplest form, the algorithm used to determine the Bragg wavelength on the measured spectrum works as follows: for each simulation, the center of mass of the reflection spectum (i.e., with spectral-shadowing) of the FBG under test is calculated. Then, the wavelength which corresponds to the center of mass of the spectrum is deduced. This wavelength is our demodulated Bragg wavelength. The difference between the measured and imposed temperature is evaluated as an error value denoted $\varepsilon$. This algorithm will be called as Center of Mass Algorithm in the rest of the text. The deteriorations on the reflection spectrum as observed in Fig. 8(a) result in an inevitable error $(\varepsilon)$ on the measured temperature. Simulations were undertaken to estimate the error due to spectral-shadowing effect on the demodulated wavelength. In these simulations, we implemented a concatenation of $N$ FBGs

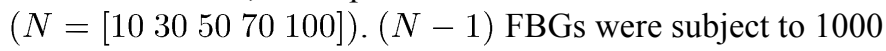
random temperature profiles (due to fabrication process and 

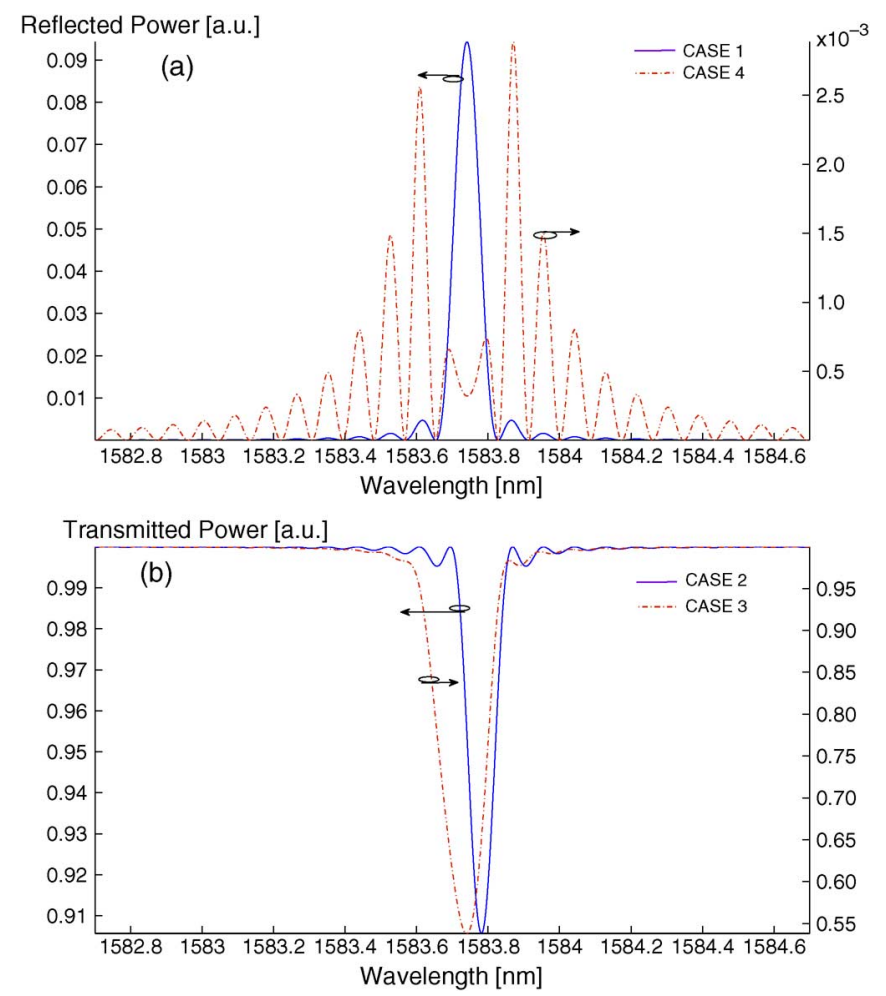

Fig. 8. (a) Case 1: light signal reflected by FBG1, at the input of the concatenation and Case 4: light signal reflected by FBG \#50 at the input of the concatenation. (b) Case 2: light signal transmitted through FBG1 and Case 3: light signal transmitted through FBG \#25.

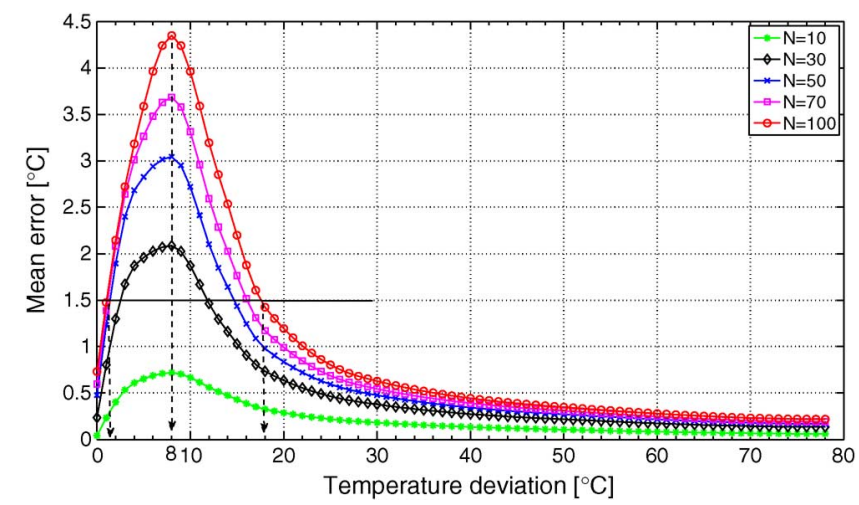

Fig. 9. Error on the $N^{t h}$ FBG due to spectral-shadowing crosstalk as a function of temperature deviation. Temperature deviation $=$ temperature to be measured on the FBG under test - mean temperature of all downstream FBGs.

modeled by a Gaussian distribution around $1583.7 \mathrm{~nm}$ with a standard deviation of $50 \mathrm{pm}$ and the last FBG (FBG \#N) was kept at fixed temperature. Center of Mass Algorithm was used to determine the Bragg wavelength as explained before.

The mean value and standard deviation of the error (between the imposed and the demodulated temperature) for different values of $N$ is reported in Table II when FBG under test (last FBG) is at $40^{\circ} \mathrm{C}$. As expected, the bigger the number of concatenated gratings, the larger the error. The mean error, computed on 1000 samples, is of maximum $14.2 \mathrm{pm}$, which corresponds to a temperature error of about $1.4{ }^{\circ} \mathrm{C}$.

The mean error value depends not only on the number of gratings but also on the offset (deviation) between the temperature

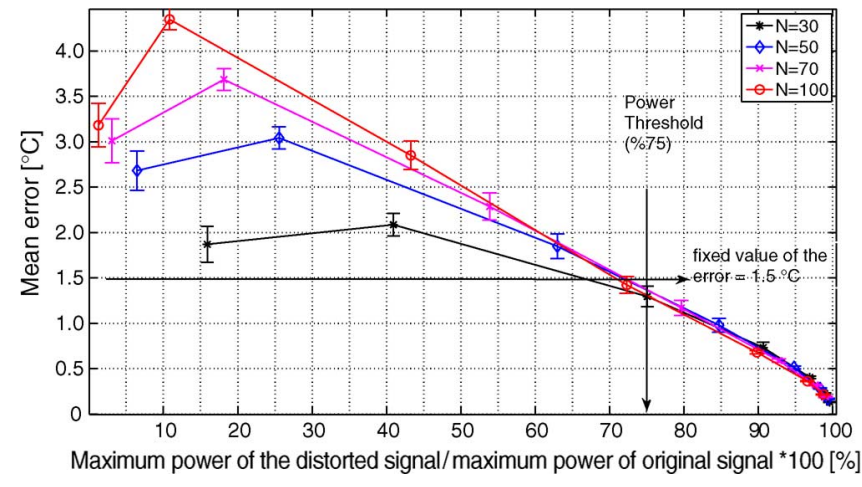

Fig. 10. Relationship between the mean error and the maximum power of the reflection spectrum. (The downstream FBGs are modeled by a Gaussian distribution around $1583.7 \mathrm{~nm}$ with a standard deviation of $50 \mathrm{pm}$ ). Error bars represent the standard deviation of the error over 1000 simulations.

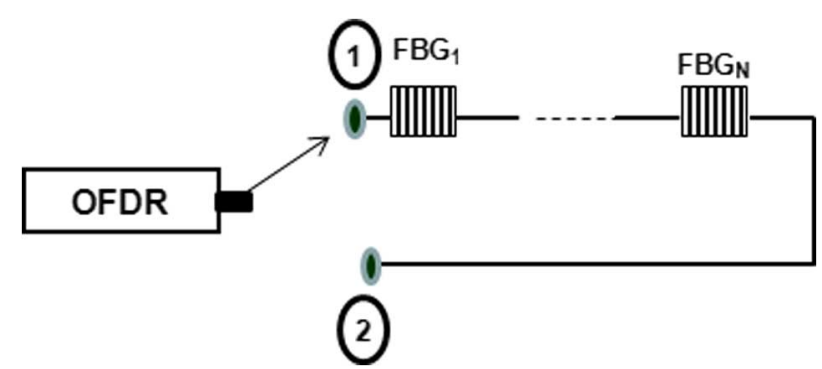

Fig. 11. Enhanced setup (measurements from two inputs by using an optical switch) that can be used to decrease the measurement errors due to spectralshadowing crosstalk.

to be measured (temperature of the FBG under test) and the mean temperature of the downstream gratings (other gratings located before the FBG under test). The temperature deviation is equal to $18{ }^{\circ} \mathrm{C}(40-22=18)$ for the results represented in Table II. Fig. 9 represents the relationship between the mean error and the temperature deviation for different numbers of cascaded FBGs. Small deviations around $5{ }^{\circ} \mathrm{C}$ up to $20^{\circ} \mathrm{C}$ induces high error values (up to $4.5^{\circ} \mathrm{C}$ for $N=100$ ) as the spectra of the downstream FBGs overlap with the spectrum of the FBG under test. Starting from $18{ }^{\circ} \mathrm{C}$ of deviation, the error becomes smaller than $1.5^{\circ} \mathrm{C}$.

In order to avoid high error values on the measured temperature occurring in the problematic region $\left[1^{\circ} \mathrm{C}-18^{\circ}\right]$ shown in Fig. 9, we adopted a new measurement method using an enhanced algorithm that detects the cases where the error is higher than a fixed value. To do so, the maximum power in the main lobe of the reflection spectrum of the distorted signal is used. It is shown in Fig. 10 that, the higher the mean error, the smaller the maximum power of the reflection spectrum (simulations on 1000 samples with the conditions defined before). This is because the reflection spectrum of the FBG under test is multiplied by the transmission spectra of all downstream FBGs giving rise to a power decrease on the detected spectrum. One should note, however, that, in Fig. 10 each data point on the figure is obtained for a given temperature deviation. From left to right on the figure, the points are obtained for increasing temperature deviations. Therefore, the small enhancement observed in the beginning is related to the fact that the distorted spectrum is a bit more symmetrical in the very beginning than that of the second 


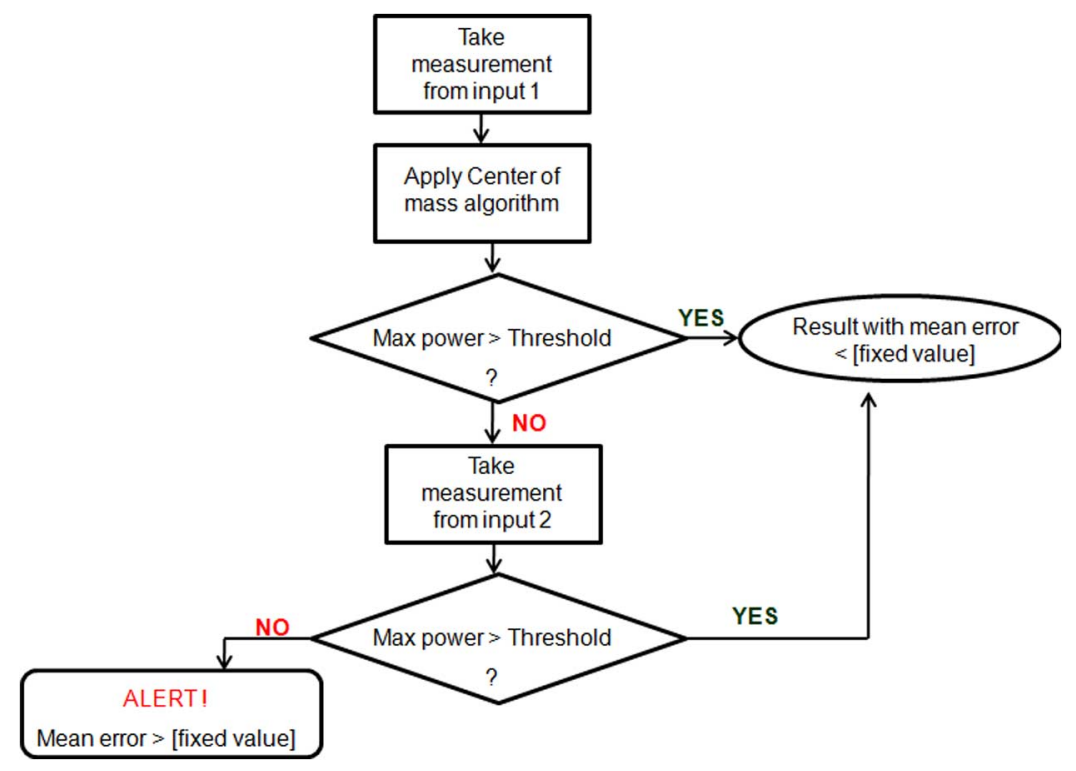

Fig. 12. Flowchart of the enhanced algorithm applied for each FBG.

data point. As a result, the center of mass algorithm works slightly better, but still giving a mean error value bigger than the threshold value $\left(1.5^{\circ} \mathrm{C}\right)$.

By using Fig. 10, one can determine a threshold value for the maximum power of the measured spectrum so that below this threshold, the mean error is bigger than a predetermined value. In our study we fixed the critical mean temperature error value to $1.5^{\circ} \mathrm{C}$, which is equal to the repeatability of our measurement setup (see paragraph 3.2).

In addition to the power threshold used in our new approach, the measurement setup is also slightly modified so that it is possible to take measurements from two extremes of the concatenation as shown in Fig. 11. For a given measurement (taken from input 1 in Fig. 11 and for each FBG), the power threshold is first checked. If the measured power is lower than the threshold value (in our case, $75 \%$ of the original power), the measurement is repeated from the other direction of the sensor (input 2 in Fig. 11). If the error is still above the threshold, an alarm is given. Indeed, the Bragg wavelengths of the FBGs which are highly affected by the spectral-shadowing crosstalk (far-end of the concatenation from input 1) can be correctly determined from the input 2 . These steps are represented in Fig. 12.

We evaluated the enhanced adaptive algorithm on the worst case shown in Fig. 12, where there are 100 FBGs in the concatenation. The temperature of the FBG under test is equal to $30{ }^{\circ} \mathrm{C}$ which gives a temperature deviation of $8{ }^{\circ} \mathrm{C}(30-22=8)$, peak point in Fig. 9. The power threshold is fixed to $75 \%$ of the maximum power of the original reflection spectrum as explained before. We then calculated the mean error on the demodulated temperature on 1000 simulations by using the enhanced algorithm (temperature profile of all the FBGs different than the FBG under test are modeled by a Gaussian distribution around $1583.7 \mathrm{~nm}$ with a standard deviation of $50 \mathrm{pm}$ ). In the following simulations, four cases have been investigated. For each of them a different FBG has been considered as the FBG under test. Cases 1, 2, 3 and 4, correspond to the cases where the FBG under test is the FBG $\backslash \# 60$, FBG \#70, FBG \#80, and FBG \#90 respectively as schematically shown in Fig. 13.

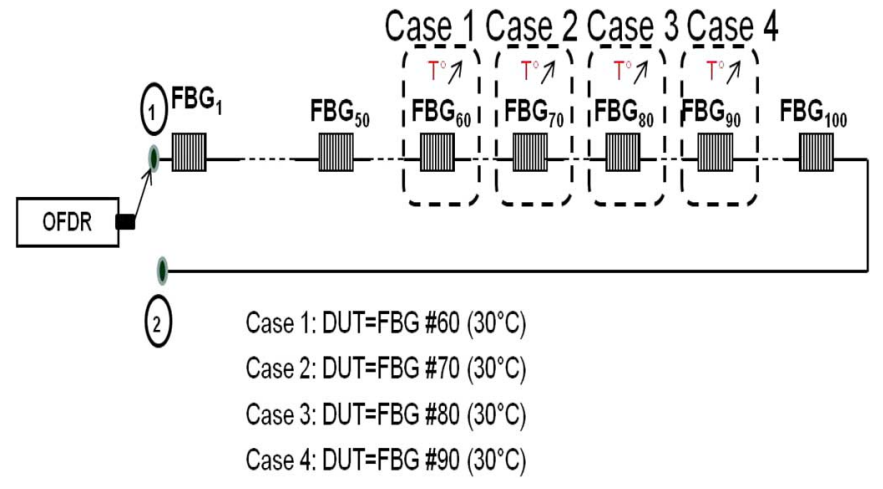

Fig. 13. Four cases used for the evaluation of the enhancement obtained by the enhanced algorithm.

TABLE III

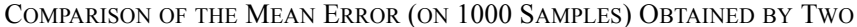
DifFERENT METHODS. FBG UNDER TEST IS AT $30{ }^{\circ} \mathrm{C}$ (WORST CASE IN Fig. 9). TeMPERATURE PROFILE OF THE FBGS DifFERENT THAN THE FBG UNDER TEST ARE MODELEd BY a GaUSSIAN Distribution ARound $1583.7 \mathrm{~nm}$ With a STANDARD DEVIATION OF 50 pm

\begin{tabular}{|l||c|l|}
\hline Error $\left[{ }^{\circ} \mathrm{C}\right]$ & Simple center of mass algorithm & Enhanced method \\
\hline \hline Case 1 & 3.4 & 2.6 (Alarm!) \\
\hline Case 2 & 3.7 & 2.0 (Alarm!) \\
\hline Case 3 & 3.9 & $1.5(\mathrm{ok} !)$ \\
\hline Case 4 & 4.2 & $0.7(\mathrm{ok} !)$ \\
\hline
\end{tabular}

Mean errors given by our previous (Center of Mass) algorithm and the enhanced method (applying power threshold and measurements from two inputs) are compared in Table III. One can observe a small enhancement of $0.8^{\circ} \mathrm{C}$ for case 1 . In case 2 , even though there is a considerable enhancement $\left(1.7^{\circ} \mathrm{C}\right)$ in the mean error, an alarm is given as the enhanced mean error is still bigger than $1.5^{\circ} \mathrm{C}$. For the cases 3 and 4, the mean errors are reduced to acceptable values thanks to the enhanced algorithm.

Among the cases where an alarm is given, the worst case corresponding to the maximum error that occurred during the simulations (i.e., over 1000 simulations), is of about $5^{\circ} \mathrm{C}$ on the measured temperature. 


\section{CONCLUSION}

This paper presents a quasi-distributed temperature sensor based on the concatenation of identical, low-reflective FBGs working as sensing points. The optical frequency-domain reflectometry technique has been used as the interrogator scheme. The distance between concatenated gratings can be at the order of a few $\mathrm{cm}$. Limitations arise from multireflection crosstalk and spectral shadowing crosstalk on the accuracy of the measured temperature and the number of FBGs in the array. These limitations have been studied together with the practical solutions to the problems which in turn gave the overall picture of the system parameters.

Using a random (uniform) distribution of the distances between the FBGs is shown to be very effective to overcome the multireflection crosstalk. Indeed, for signal to multireflection crosstalk ratio (SMRR) better than 10, the maximum number of FBGs that can be concatenated is enhanced from about 30 (equal distance case) to about 100 by using a uniform distribution of the distance between sensing points.

The influence of the spectral-shadowing crosstalk is found to be dependent on the temperature deviation between the mean value of all downstream FBGs and the temperature to be measured on the FBG under test. By using an enhanced demodulation algorithm, the error on the measured temperature due to spectral-shadowing crosstalk has been considerably reduced (up to $3.5^{\circ} \mathrm{C}$ of enhancement).

\section{ACKNOWLEDGMENT}

The authors would like to thank to the Computer Science Department of UMONS, particularly S. Frémal for offering the code optimization process and the computational resources.

\section{REFERENCES}

[1] A. Mendez, "Fiber Bragg grating sensors: A market overview," Proc. SPIE, pp. 661905-1-661905-6, 2007.

[2] Othonos and Kalli, Fiber Bragg Grating Sensors Fibre Bragg Gratings: Fundamentals and Applications Telecommunications and Sensing. Norwood, MA: Artech House, 1999.

[3] Y.-L. Lo and S.-H. Xu, "New sensing mechanisms of an optical time doma reflectometry with fiber Bragg gratings," presented at the OSA Tech. Dig., 2006, TuE4.

[4] A. D. Kersey, M. A. Davis, and T. Tsai, Japan Society of Applied Physics, "Fiber optic Bragg grating stra sensor with direct reflectometric interrogation," in Proc. 11th Int. Conf. Optical Fiber Sensors, Sapporo, Japan, 1996, pp. 634-637.

[5] C. Crunelle, C. Caucheteur, M. Wuilpart, and P. Mégret, "Quasi-distributed temperature sensor combining Fibre Bragg Gratings and temporal reflectometry technique interrogation," Opt. Lasers Eng., vol. 47, pp. 415-418, 2009.

[6] C. Crunelle, C. Caucheteur, M. Wuilpart, and P. Mégret, "Original interrogation system for a quasi-distributed FBG-based temperature sensor with fast demodulation technique,"Sens. Actuators A, vol. 150, pp. 192-198, 2009.

[7] J. Zheng, "Analysis of optical frequency-modulated continuous-wave interference," Appl. Opt., vol. 43, pp. 4189-4198, 2004.
[8] B. A. Childers, M. E. Froggatt, S. G. Allison, T. C. Moore, D. A. Hare, C. F. Batten, and D. C. Jegley, "Use of 3000 Bragg grating sensors distributed on four eight-meter optical fibers during static load tests of a composite structure," in Proc. SPIE, 2001, vol. 4332, pp. 133-142.

[9] Y. Shinoda, I. Ogura, Y. Ono, S. Kaneko, and T. Higo, "Fundamental experiment of multiple-point measurement for stra by fiber Bragg gratings using optical frequency sweeping," in Proc. Int. Joint Conf. SICEICASE, 2006, vol. 4332, pp. 1672-1675.

[10] A. M. Abdi, S. Suzuki, A. Schülzgen, and A. R. Kost, "Modelling, design, fabrication, testing of a fiber Bragg grating stra sensor array," Appl. Opt., vol. 46, pp. 2563-2574, 2007.

[11] K. Yüksel, M. Wuilpart, V. Moeyaert, and P. Mégret, "Novel monitoring technique for passive optical networks based on optical frequency doma reflectometry and fiber bragg gratings," J. Opt. Commun. Netw., vol. 2, pp. 463-468, 2010.

[12] R. G. Duncan, B. A. Childers, D. K. Gifford, D. E. Pettit, A. W. Hickson, and T. L. Brown, "Distributed sensing technique for test article damage detection and monitoring," in Proc. SPIE, 2002, vol. 5050 , pp. 367-375.

[13] A. D. Kersey, M. A. Davis, H. J. Patrick, M. LeBlanc, K. P. Koo, C. G. Askins, M. A. Putnam, and E. J. Friebele, "Fiber grating sensors," J. Lightw. Technol., vol. 15, pp. 1442-1463, 1997.

Kivilcim Yüksel (M'10) received the M.S. degree from the Electronics Engineering Department, Ege University, Izmir, Turkey, in 2000, and the Postgraduate Advanced degree and the Ph.D. degree in fiber optics from the Faculte Polytechnique de Mons (FPMs), University of Mons, Mons, Belgium, in 2006 and 2011, respectively.

Between 2002 and 2005, she was with Multitel asbl (Mons, Belgium) where she was involved in a project dedicated to monitoring methods based on optical time domain reflectometry (OTDR) technique for point-to-multipoint optical access networks. She then joined the Electromagnetism and Telecommunications Department of the FPMs in 2005, where she was a teaching assistant

Véronique Moeyaert (M'97) received the $\mathrm{Ph} . \mathrm{D}$. degree in applied sciences from the Faculte Polytechnique de Mons (FPMs), University of Mons, Mons, Belgium, in 2002.

Since 2005, she has been an Associate Professor of telecommunications at the Engineering Faculty, University of Mons. Her main interests are in the physical layer characterization of bi-directional communications over hybrid fibre optic and coaxial (HFC) CATV networks and in digital systems performance estimation in non Gaussian environments.

Dr. Moeyaert is a member of the TC209 CENELEC committee.

Patrice Mégret (M'87) was born in 1964. He received the Ph.D. degree in applied sciences from the Faculte Polytechnique de Mons (FPMs), University of Mons, Mons, Belgium, in 1993.

$\mathrm{He}$ is presently Head of the Electromagnetism and Telecommunication Department, FPMs, University of Mons. His field of interest is related to photonic technology with emphasis on system metrology. He is the author or coauthor of more than 300 publications in journals and conference proceedings.

Dr. Mégret was president of the IEEE LEOS Benelux Chapter from 2003 to 2005 and is an associate member of URSI.

Marc Wuilpart (M'03) received the Ph.D. degree in applied sciences from the Faculte Polytechnique de Mons (FPMs), University of Mons, Mons, Belgium, in 2003 .

He is currently an Associate Professor in classical and optical telecommunications at FPMs. His main research interests are in spatially distributed measurements in optical fibers and in studying the polarization properties of optical devices for telecommunication and sensing applications. He is the author and coauthor of one book chapter and more than 60 journal and conference papers. 